\title{
Las raíces de la pedagogía crítica y el diseño curricular crítico en Costa Rica: 1910-1948
}

\author{
Toruño Arguedas, César \\ Las raíces de la pedagogía crítica y el diseño curricular crítico en Costa Rica: 1910-1948 \\ Revista Educación, vol. 44, núm. 2, 2020 \\ Universidad de Costa Rica, Costa Rica \\ Disponible en: http://www.redalyc.org/articulo.oa?id=44062184002 \\ DOI: https://doi.org/10.15517/revedu.v44i2.39787
}

Esta obra está bajo una Licencia Creative Commons Atribución-NoComercial-SinDerivar 3.0 Internacional. 


\section{Las raíces de la pedagogía crítica y el diseño curricular crítico en Costa Rica:}

\section{$1910-1948$}

\section{Roots of Critical Education and Critical Curriculum Design in Costa Rica: 1910-1948}

César Toruño Arguedas

Universidad Técnica Nacional, Costa Rica

cesartoruno@gmail.com

iD http://orcid.org/0000-0003-0476-7893
DOI: https://doi.org/10.15517/revedu.v44i2.39787

Redalyc: http://www.redalyc.org/articulo.oa?id=44062184002

Recepción: 17 Diciembre 2019

Aprobación: 31 Enero 2020

\section{RESUMEN:}

El ensayo desarrolla un análisis de los principios conceptuales y demandas de transformación elaborados por intelectuales pedagógicos de los movimientos progresistas costarricenses ligados con el Partido Comunista, específicamente durante la primera mitad del siglo XX como el antecedente inmediato del surgimiento de una pedagogía crítica y un diseño curricular alternativo o crítico en el contexto costarricense. Para elaborar este estudio se establece la caracterización de intelectuales y el análisis de sus propuestas desde los ejes pedagogía, sociedad y reformas educativas, educación-centro educativo, los roles asignados a las personas docentes y la transformación de la enseñanza-aprendizaje; estos ejes permiten la visibilización de las interacciones argumentativas de una escuela de pensamiento costarricense vinculable con el origen de la pedagogía crítica en nuestro país.

Palabras clave: Currículo, Currículo Crítico, Pedagogía Crítica, Progresismo Pedagógico, Costa Rica.

\section{Abstract:}

This study looks at conceptual principles and transformational requirements developed by pedagogical intellectuals from the Costa Rican Progressive Movement linked with the Communist Party, specifically during the first half of the $20^{\text {th }}$ Century as an immediate precursor to critical pedagogy and alternative or critical curriculum design in the Costa Rican context. Preparation for this analysis is based on characterizing intellectuals and analysis of their proposals from a perspective involving pedagogy, society and educational reform, education and schools, the assigned roles of teachers and transformation of teaching-learning. These perspectives shed light on argumentative interactions from a Costa Rican school of thought considered to be the origin of the country's critical pedagogy.

KeYwords: Curriculum, Critic Curriculum, Critical Pedagogy, Pedagogical progressivism, Costa Rica.

\section{INTRODUCCIÓN}

Al finalizar el siglo XIX, Costa Rica es escenario de la consolidación del modelo liberal y agrario capitalista, y el desarrollo de un grupo de intelectuales y movimientos sociales críticos con el modelo económico, social y cultural defendido por la burguesía costarricense.

Como parte de los movimientos contestatarios, entre las décadas de 1910 y 1948, es posible identificar un movimiento de educadores quienes participaron activamente de grupos de reflexión-acción para la ejecución de transformaciones educativas en la contextualización curricular; insertar visiones críticas y científicas a la estructura del currículo oficial tradicionalmente conservador (Molina, 2000) y desarrollar propuestas educativas desde una lectura del comunismo costarricense.

A partir de la pregunta generadora ¿es posible identificar elementos de la pedagogía crítica en los discursos y propuestas políticas de Joaquín García Monge, Omar Dengo, Carmen Lyra, Carlos Luis Sáenz, Arnoldo Ferreto y Luisa González? El presente ensayo sistematiza un análisis de las producciones literarias, artículos y ensayos publicados en Periódico Trabajo (Partido Comunista), el quincenario de pedagogía El Maestro, y 
fuentes secundarias, desarrollado a partir de la utilización de fichas para el análisis de contenido y un método histórico para el análisis interno y externo de los documentos.

La articulación de la información obtenida ha sido organizada, en el presente documento, en los apartados pedagogía y sociedad, educación y centro educativo, roles docente-estudiante y contextualización curricularenseñanza, aprendizaje, evaluación y conocimiento. Estos apartados y la interacción de sus elementos conceptuales dentro del pensamiento pedagógico los educadores y las educadoras en estudio, permite establecer las características de un currículum alternativo o crítico y, por tanto, las raíces de una pedagogía crítica costarricense.

\section{Pedagogía, sociedad y reformas educativas}

\section{Al iniciar el siglo XX, Omar Dengo (2009) afirmaba que}

en todas las épocas, pero sobre todo en la presente, el problema social es problema de educación, por excelencia [e indicaba que] no hay posibilidad de deslindar con rigor los dos aspectos sociales de la escuela que es, al mismo tiempo, factor y efecto del progreso (p. 343).

Estas premisas resultan fundamentales para comprender las reivindicaciones de la pedagogía crítica costarricense durante la primera mitad del siglo XX, esto con el objetivo de efectuar una ruptura con la narración dominante sobre la neutralidad del sistema educativo y la supuesta única misión de formar académicamente a los futuros ciudadanos, al respecto el periódico Trabajo publicó el artículo Los hijos de los trabajadores denunciando que

hasta ahora a la pedagogía le ha resultado más cómodo ser indiferente a los problemas sociales desde el punto de vista político y económico; y la escuela como institución burguesa que es, aparenta resolver los problemas por medio de la filantropía, y limita tus actividades a la prédica de virtudes burguesas como el ahorro, la caridad, etc. (Anónimo, 13 de marzo 1932, p. 2).

El posicionamiento adquiere relevancia por cuanto establece la asociación de la escuela como institución burguesa un elemento clave en las posturas de la pedagogía crítica bajo el enfoque marxista tradicional y establece la limitante del enfoque asistencialista como mecanismo para solucionar los problemas sociales. Este último con la característica de construir estadísticas y datos que no aportaban soluciones en las realidades de los desposeídos pues "cientos y miles de los niños anotados en esas fichas guardadas en esos archivos seguirán pasando hambre y durmiendo en camas compartidas con otras tres o cuatro personas, en casas malsanas" (Anónimo, 7 de octubre de 1934, p. 1).

En línea con las anteriores reivindicaciones, Lyra (28 de julio de 1935) recordaba:

En una ocasión recibí de un niño una lección muy buena. Yo les decía: ojalá se acuesten a las siete de la noche, que duerman bien abrigados, pero con la ventana abierta, y cada uno en su camita. Un chiquillo me replicó: -En casa no hay ventana y como la casa es muy chiquita, solo cabe una cama y en ella dormimos mi mamá, mis cuatro hermanos y yo...

Pero los maestros que saben muchas cosas de castellano, de matemáticas, de geografía y de historia, dicen que en Costa Rica no hay problemas económicos ni sociales (p. 3).

En esta reflexión, Carmen Lyra establece la división entre las personas pedagogas tradicionales y aquellas afiliadas al comunismo, los primeros limitados a la reproducción de conocimientos descontextualizados o negación de realidades (claramente reflejado en las clases de higiene para estudiantes en extrema pobreza) y la persona educadora crítica capaz de hablar con sus estudiantes, entender sus contextos y comprender los problemas económicos y sociales.

La reivindicación de la escuela en un contexto social, así como la necesidad de asumir y transformar esa realidad, es una idea reiterada en los diversos textos de los autores en estudio, un referente es el cuento Niños, parte de la obra Bananos y Hombres, en la cual Carmen Lyra describe la difícil situación económica y social 
de los niños en la zona atlántica (Lyra, 1977). En el cuento Lucía, Lyra describe su visita a la casa de una de sus estudiantes que se había ausentado por tres días, indica que el barrio es

... un basurero, y en un rincón la casa llena de chuicas sucios y entre los chuicas la pobre ardiendo en calentura. Y solita, al cuidado del buen corazón de los vecinos. Sobre el asiento de una silla renca, un jarro de lata con agua por si le da sed. La madre se va al trabajo desde las siete de la mañana; vuelve a las once a hacer el almuerzo y a tragarse los bocados porque a las doce tiene que estar en la cervecería, y después hasta las cinco, si no es que se le presenta en el camino algún amorío. (Lyra, 1977, p. 133).

Con la anterior argumentación, Lyra reitera la exclusión (Lyra, 28 de julio de 1935) promovida por las clases dominantes y las desigualdades, en el marco de los impactos para los niños y las niñas. Estas reflexionesdenuncias adquieren relevancia por darse en una sociedad con narraciones legitimadoras que pretendían caracterizar a Costa Rica como una sociedad igualitaria, carente de acumulación de la riqueza y con más maestros que soldados; esto último cuestionado por el propio Dengo (2009) al afirmar que "cuando los periódicos, en los editoriales de réclame (sic) patriotero, repiten... que el país tiene más maestros que soldados, encubren la tremenda verdad de que tiene más analfabetos que proyectiles” (p. 332).

La posición de Lyra se consolida en el Congreso Pedagógico desarrollado en Costa Rica en 1934, evento en el cual participan los pedagogos comunistas; el periódico Trabajo reseña la posición de Luisa González en el artículo El congreso pedagógico (Anónimo, 7 de octubre de 1934) y la reiteración de la relación intrínseca entre reforma educacional y condiciones económicas-ambientales. Elemento que sería reiterado el 15 de septiembre de 1940 por Carlos Luis Sáenz (15 de septiembre de 1940) al indicar,

Resolviendo en primer término la economía del hogar costarricense y para resolverla hay que resolver el problema de nuestra economía nacional. Mientras tanto haya miseria en la masa del pueblo, la educación será por ese mismo hecho privilegio de los adinerados, por tanto, perderá un $99 \%$ de su espíritu democrático (p. 7).

La agenda de lucha se extendió a debates sobre los contenidos y funciones de la educación, por ejemplo, en Trabajo se destaca las conferencias de Luisa González sobre sexualidad, indicando que González "ha querido enseñarles a los trabajadores de ambos sexos -y lo está logrando- que deben ser ellos quienes rompan de primeros esos velos de falsa pudicia con que se pretende ocultar a los chiquillos los misterios de la vida sexual" (Anónimo, 10 de octubre de 1931, p. 4). La inclusión de la sexualidad en el discurso de los pedagogos progresistas fue usada por los conservadores para indicar que González, Sáenz y Lyra irían a "predicar el amor libre, el aborto y la disolución de la familia" (Lyra, 27 de mayo de 1939, p. 4).

En respuesta a los ataques conservadores, líderes de la pedagogía crítica consolidaron una agenda educativa desde el Partido Comunista, la primera de ellas en la edición del 13 de marzo de 1932, bajo el título de Programa mínimo del Partido Comunista de Costa Rica, el cual indicaba que la política educativa se basaría en:

Reforma completa de las leyes de educación de acuerdo con los siguientes principios fundamentales: a) preferente atención a la educación pre escolar (casas cunas, escuelas para niños de 2 a 4 años, Kindergartens, escuelas maternales); b) el trabajo como eje alrededor del cual girarán las actividades de la escuela primaria y secundaria (interés especial para los trabajos manuales y agrícolas); c) educación gratuita en todas sus fases y obligatoria en las necesarias para obtener ciudadanos libres y conscientes (Anónimo, 13 de marzo de 1932a, p. 2).

La reforma (reiterada literalmente el 14 de junio de 1941 con el mismo título) es progresista en el tanto establece la necesidad de la educación preescolar con una agenda de acceso vinculación del sistema educativo y el trabajo, lo cual es una ruptura con la tradicional narración de dicha preocupación exclusivamente por el sector dominante (capitalista) y la demanda de una educación gratuita y obligatoria en todos los niveles.

La segunda agenda de reforma educativa se presenta el 4 de febrero de 1934, con el título Conquistas inmediatas que para los maestros propone el Bloque de Obreros y Campesinos, se indica como objetivos:

18- Establecimiento de un Consejo Nacional de Educación que opere la independización del magisterio del poder Ejecutivo. 
19- Legislación protectora de los maestros rurales en el sentido de que sean las Juntas de Educación quienes sufraguen los gastos de los maestros que trabajen en lugares diferentes de aquellos en donde bien; y que los gastos de viático cuando lo requiera su traslado corran de cuenta del Estado.

20-Reforma a la ley de pensiones para que estas se concedan al terminar los años de servicio reglamentario aun cuando los maestros no hayan cumplido los 50 años de edad que ahora se exigen.

21- Pago del sueldo completo a los maestros enfermos. Pensiones y seguros de vida de los maestros a cargo del Estado (Anónimo, 4 de febrero de 1934, p. 4).

Las medidas propuestas, centradas en los educadores y las educadoras, aspiraban a liberar al sistema educativo de la influencia política (creación del Consejo Nacional de Educación) y la respectiva persecución a docentes. Junto a esta demanda, se establece la necesidad de asegurar la independencia del magisterio con respecto al poder de la oligarquía cafetalera y gamonales en las zonas rurales y, por último, las demandas de protección laboral para los docentes. Todos estos temas entendibles no solo por las realidades del sistema educativo sino, además, por la necesidad del Partido Comunista para ampliar su base de apoyo dentro del magisterio de cara a las elecciones.

La tercera agenda educativa se presenta el 15 de septiembre de 1938 bajo el título Política del Partido Comunista: Lucha por la ampliación de la Democracia Costarricense, los puntos pedagógicos indican:

Política Educacional

ARTÍCULO $15^{\circ}$ - Creación de un Consejo Superior de Educación Pública, que dé unidad y orientación a la Educación Nacional, de acuerdo con las peculiaridades de nuestra economía, lo mismo q' (sic) tomando en consideración la necesidad de capacitar un sector de los educandos para los estudios superiores universitarios.

ARTÍCULO $16^{\circ}$ - Enseñanza secundaria gratuita y costeada por el Estado. El Estado mantendrá un servicio de becas en el extranjero para estudiantes sobresalientes de los Colegios de Segunda Enseñanza.

ARTÍCULO $17^{\circ}$ - Libertad de cátedra, derecho de asociación e inamovilidad para el Magisterio Nacional.

ARTÍCULO $18^{\circ}$ - Para que la Enseñanza Primaria sea efectivamente gratuita, el Estado proporcionará, a la población escolar pobre, todos los útiles que demande su educación, lo mismo que la alimentación indispensable cuando los hogares no estén en condiciones de proporcionarla.

ARTÍCULO $19^{\circ}$ - Plan científico de construcciones escolares, a fin de que no se quede el niño sin matrícula, por falta de espacio en los edificios escolares. Abolición del sistema de horarios alternos.

ARTÍCULO $20^{\circ}$ - Reajuste de los sueldos del Personal Docente, los cuales, en la actualidad, no están de acuerdo con las necesidades honestas de un ser civilizado; lucha por el establecimiento del carnet docente y estudiantil. Lucha por la exoneración del aforo a todos los libros, cuya fabricación sea imposible en el país (Anónimo, 15 de septiembre de 1938, p.5).

Esta tercera agenda educativa del Partido Comunista demuestra una mayor madurez política y pedagógica del Partido con respecto a las necesidades del país y la capacidad de síntesis para expandir el mensaje entre el gremio docente y familias. El Consejo Nacional de Educación mencionado en sus primeras agendas como organismo que permitiría velar por la estabilidad laboral de los docentes adquiere relevancia como institución para organizar y orientar el sistema educativo; se incrementa la demanda de acceso y gratuidad de la educación (primaria y secundaria), un plan de construcciones y reivindicaciones laborales (estabilidad, sueldos, pensiones y otros).

En 1942, el Partido Comunista presenta su cuarta agenda educativa, la más amplia presentada hasta el momento por el partido. Para estas elecciones de medio periodo, esta agrupación política se presentaba como fuerza emergente y coaligada al Partido Republicano, impulsora de las reformas sociales, cambio de nombre, acercamiento con la iglesia católica, una estructura organizativa en crecimiento, el Periódico Trabajo expandía su alcance (más de 20 mil ejemplares por edición) y consolidaba las células en los cantones de todo el país. En este contexto, se explica la profundización de sus temas educativos, a saber:

Política Educacional:

La educación nacional está al servicio de nuestra democracia. Por tanto, da igual de oportunidades a todos los individuos de la generación joven. El Estado ha de ser el principal agente de la educación nacional y ha de servirse de ella como medio télico (sic) para el desarrollo económico y cultural de nuestra democracia.

Concretamente, se señalan las siguientes tareas a realizar en el campo de la política educacional del país: 
a)- Creación de un Consejo Superior de Enseñanza Pública que unifique y oriente la educación nacional, de acuerdo con las peculiaridades de nuestra economía y al mismo tiempo tomando en consideración la necesidad de capacitar un sector de educandos para los estudios superiores universitarios.

b)-Ampliación de la escuela primaria con un Sétimo grado, con carácter facultativo para la enseñanza de las manualidades y de la educación cívica (varones) y de la puericultura y educación doméstica (señoritas).

c)- Implantación y desarrollo del tipo de escuela granja como institución educativa deseable en nuestro medio rural.

d)- Creación de escuelas de Artes y Oficios y de Bellas Artes con matrículas libres para estudiantes pobres, sostenidas por el Estado.

e)- Para que la enseñanza nacional sea efectivamente gratuita, el Estado proporcionará a la población escolar pobre, todos los útiles que demandan su educación, lo mismo que la alimentación indispensable, ropa, calzado, recreaciones y servicio médico cuando los hogares no estén en condiciones de proporcionar tales servicios.

g)- (sic) Establecimiento de intercambio de maestros, profesores y estudiantes con los otros países latinoamericanos y con los Estados Unidos.

h)- Tanto en la educación oficial como en la privada, solo podrán ejercer la enseñanza los educadores de reconocida ideología favorable al mantenimiento y desarrollo de las libertades democráticas.

j)- Derecho de asociación e inamovilidad para el magisterio nacional como base indispensable de seguridad, de defensa y de progreso gremial.

k)- Fomento y ampliación de escuela para anormales.

1)-Creación de un gabinete de investigaciones psico-pedagógicas.

m)- Reajuste de los sueldos del Personal Docente, los cuales en la actualidad no están de acuerdo con las necesidades honestas de un ser civilizado. Establecimiento del Carnet Docente y estudiantil. Rebaja en los transportes nacionales para el personal docente y para los estudiantes. Exoneración de aforos para libros e implementación de laboratorios con destino a las instituciones de educación cuya elaboración no sea posible en el país.

Para ampliación de la cultura de maestros y profesores, servicios de becas internas y externas; conferencias culturales y establecimiento de una radiofusora (sic) a cargo del Consejo de Educación Pública conectada con los países más avanzados del mundo.

n)-Intensificación del sistema de Patronatos Escolares como medio de vincular a la obra educativa a las personas más serviciales del vecindario o de la comunidad (Anónimo, 10 de enero de 1942, p. 4).

De la propuesta, se desprende la reiteración de la creación del Consejo Superior de Educación Pública (punto a) como mecanismo para asegurar la independencia política del sistema educativo, por su parte el punto $b$ incorpora la ampliación de la escuela con una división sexista de la formación, esto último comprensible en la lógica de la sociedad patriarcal. Los puntos $c y d$ demuestran la preocupación por establecer un sistema educativo pertinente para las demandas laborales en una sociedad agraria y de artesanos, con núcleos de formación artística para ampliar las posibilidades culturales de las clases desposeídas, esto último puede ser vinculado a la historia de vida de Luisa González y Carmen Lyra como docentes y artistas.

El punto $e$ profundiza la reivindicación histórica del comunismo con respecto a la educación gratuita ampliado a elementos de útiles, alimentación, matrícula, ropa, calzado, recreaciones y servicio médico. Al respecto se debe destacar que el 20 de enero de 1945, bajo el título Enseñanza Secundaria gratuita para estudiantes pobres, (Anónimo, 1945) se comunica la aprobación de la reforma al Código de Educación para establecer la enseñanza gratuita, uno de los primeros logros de la agenda educativa defendida por los pedagogos comunistas.

Por su parte, el punto $g$ establece los intercambios de docentes y estudiantes con otros países latinoamericanos y con Estados Unidos, lo cual podría vincularse a la experiencia de intercambio que tuvo Carmen Lyra (beca a Europa) y la importancia de estas actividades para la ruptura de mentalidades de quienes asisten.

Los puntos $h, j$ y $m$ se vinculan a las demandas históricas del Partido Comunista sobre la estabilidad laboral con la inclusión de apartados como revisión de la ideología de los educadores relacionado con el mantenimiento y desarrollo de las libertades democráticas, un llamado de atención implícito al grupo políticopedagógico ligado con el cortesismo y lo que el Partido Comunista denominaba fascistas costarricenses y, por último, solicitud de rebaja en transporte para docentes y estudiantes así como en los libros e implementos de laboratorio; estas últimas demandas están vinculadas a las necesidades directas de estudiantes y docentes, 
factor importante en una elección intermedia sucedida en plena crisis económica por la Segunda Guerra Mundial.

La cuarta agenda pedagógica del Partido Comunista innova con la inclusión del Fomento y ampliación de escuela para anormales (punto $\mathrm{k}$ ) y la Creación de un gabinete de investigaciones psico-pedagógicas (punto 1), lo cual demuestra un perfeccionamiento y especialización mayor de la agenda pedagógica, posiblemente influida por Carlos Luis Sáenz, quien durante el primer quinquenio de 1940 profundizaría las temáticas pedagógicas difundidas en Trabajo. Por último, el punto $n$ incorpora la demanda de Patronatos Escolares para crear enlaces permanentes entre la escuela y la comunidad.

Un año después, el Partido Comunista presentaría una quinta agenda educativa, en el marco del cambio de nombre y reorientación de la estrategia política, integrada en el Programa de Vanguardia Popular, el cual reitera los elementos de la cuarta agenda e incluye los siguientes elementos:

Política Educacional:

...d)- (sic) Enseñanza Secundaria gratuita para estudiantes pobres y costeada por el Estado. El Estado mantendrá un servicio de becas en nuestra Universidad o en el extranjero, para estudiantes pobres sobresalientes en los Colegios de Segunda Enseñanza....

j)- Revisión del problema de la delincuencia infantil y creación de Tribunales de Menores científicamente organizados... (Anónimo, 26 de junio de1943, p. 3).

Con esta quinta agenda, el Partido Comunista (ahora denominado Vanguardia Popular) reitera las propuestas elaboradas previamente e incluye el apartado de becas para los estudiantes pobres que logren terminar la secundaria para que, como parte de la reivindicación de la ampliación de la cultura de la nación y la política de supresión de desigualdades, pudiesen ingresar a la Universidad de Costa Rica (de reciente creación) o una universidad extranjera. Destaca la inclusión de la temática de la delincuencia infantil y tribunales de menores, en el tanto no había sido referenciado en los 12 años previos de divulgación del Periódico Trabajo. Un año después, el partido presentaría la sexta agenda educativa bajo el título Programa del Bloque de Victoria, la cual mantendría todos los elementos mencionados en la agenda anterior con la inclusión del punto o "Apoyo amplio a la Universidad Nacional" (Anónimo, 20 de febrero de 1946, p. 4).

La estructuración de la agenda educativa comunista es coherente con los orígenes de la pedagogía crítica en occidente, así como la premisa de "educar en el niño la rebeldía y el valor, hacerlo sensible al dolor de los compañeros no con las moralejas cursis de las lecturas escolares, sino haciendo sentir la realidad de la vida que tiene a la par" (Anónimo, 13 de marzo de 1932b, p. 4) y claramente con una perspectiva partidaria desde el comunismo dirigida a la formación de cuadros, en el tanto esos "chiquillos reemplazaran a sus padres en las filas combativas por la emancipación del proletariado, y tendrán bajo su responsabilidad la construcción pacífica de la sociedad sin clases" (Ferreto, 1934, p. 3).

\section{EDUCACIÓN Y CENTRO EDUCATIVO: INTERACCIÓN DIALÉCTICA CON LA SOCIEDAD}

Al iniciar el siglo XX, Carmen Lyra conceptualizaba la educación como "un campo abierto y no cerrado al palpitar de su corazón; algo en contacto directo con sus necesidades, no ajeno a la circulación de su sangre y directamente interesado en su salud" (Lyra, 15 de noviembre de 1927, p. 81). Este principio de interacción dialéctica guiaría las reflexiones educativas de las personas pedagogas críticas durante las décadas de 1930 y 1940.

En la década de 1930, y en el contexto de la constitución del Partido Comunista, la crisis de 1929 y ampliación de movimientos sociales, las personas pedagogas críticas costarricenses lograron construir una clara oposición intelectual y práctica a una educación que "ha cumplido bien con su deber de orientar al pueblo hacia sus conveniencias" (Anónimo, 19 de marzo de 1933, p. 3), y la cual es; 
La escuela ha venido siendo y es, ante todo, un instrumento de clase. Por más que los pedagogos burgueses traten de confundir, parapetándose tras una vaga nación de un ideal 'humano' la realidad de sus pretensiones, nosotros sabemos que la sociedad burguesa, en la práctica, se sirve de la escuela como arma, para defender sus intereses de clase y formar las mentalidades que ellos necesitan para defender sus privilegios (Ferreto, 1934, p. 3)

Este es el primer texto pedagógico publicado en Trabajo, en el cual se entremezclan los elementos de la narración clásica marxista y la visión de la educación en general. Estos posicionamientos se profundizarían durante el siguiente quinquenio motivados por lo que los comunistas caracterizaban como afiliaciones fascistas de diversos sectores de la burguesía costarricense, en este sentido en Trabajo se denunciaba que Teodoro Picado en calidad de Secretario de Educación había desarrollado "una serie de medidas de típico sabor fascista. El concepto de la disciplina cuartelaría ha tratado de imponerlo en todo momento y ante toda circunstancia" (Anónimo, 14 de abril de 1935, p. 1). El autor cierra el artículo denunciando que, "Militarismo, espionaje, persecución contra el maestro independiente y rebelde, ataque abierto contra el marxismo en toda oportunidad y en todo sitio" (Anónimo, 14 de abril de 1935, p. 1).

La persecución de la persona educadora crítica, principalmente ligada con el comunismo es un tema de asociación en la función del sistema educativo y el centro educativo, al respecto, en Trabajo se afirmaba que "los maestros y profesores que quieren conservar su puesto tienen que convertirse en instrumentos ciegos de los Ministros de Educación y jefes de la Enseñanza que velan perrunamente por los privilegios de los adinerados" (Anónimo, 30 de enero de 1938., p. 4). Otros autores establecen el papel de la educación para concientizar a la población estudiantil sobre las implicaciones del capitalismo, la creación de desigualdades y las posibilidades de transformación, al respecto, en Trabajo se publicó el 13 de marzo de 1932 el artículo Los hijos de los trabajadores, el cual indicaba:

La escuela tiene que dejar de vivir de abstracciones. Que la escuela instruya al niño para que este sepa que la producción y la distribución de la riqueza en los pueblos están en íntima relación con su estado de vida higiénico, moral e intelectual. La escuela luchará contra la injusticia social de una manera efectiva despertando en el niño el valor y la rebeldía y no como lo ha hecho hasta ahora preparando una masa de gentes conformes, pasivas e ignorantes (Anónimo, 13 de marzo de 1932b, p. 4).

Por tanto, en la primera mitad del siglo XX se constituye un grupo de intelectuales pedagogos el cual favorece la elaboración de una serie de ejes y reivindicaciones para el debate educativo en Costa Rica, asumiendo que el "problema de los fines es el problema fundamental de toda pedagogía" (Ferreto, 1934, p. 3). Este grupo paso de posiciones moderadas (Omar Dengo, Carmen Lyra y Luisa González) antes de 1930 a posiciones paulatinamente más radicales, tanto de los autores explícitos como en artículos anónimos que, para otros estudios, debe debatirse la autoría pues algunos coinciden en forma y temáticas con textos de Lyra y González. En paralelo a esta evolución, se construye un posicionamiento con respecto al educador que implementaría esta nueva educación, elemento a analizar en el siguiente apartado.

\section{EDUCADORES Y EDUCADORAS PARA LA TRANSFORMACIÓN Y LUCHA SOCIAL}

La construcción conceptual de la persona docente en el marco de la proyección educativa elaborada por los pedagogos críticos durante la primera mitad del siglo XX comparte la evolución del elemento conceptual educación y centro educativo, a saber, una primera etapa, previo a 1929, en la cual se reivindica que "Inculpar al maestro y solo al maestro, es un error monstruoso. Tanto valdría imputarles a los empleados de aduana el decrecimiento de los ingresos por importación” (Dengo, 2009, p. 340) asumiendo una vinculación indirecta con las premisas marxistas-pedagógicas. La segunda etapa se caracteriza por una asociación directa con las premisas marxistas y del comunismo a la tica (década de 1930) y, por último, una etapa de profundización de la propuesta educativa desde premisas marxistas (década de 1940) y el desplazamiento de la agenda educativa en el marco de la priorización de las reformas sociales y el contexto pre guerra civil. 
En la primera etapa, los pedagogos críticos y las pedagogas críticas utilizaron narraciones dentro de la oficialidad para incluir las premisas de transformación, un ejemplo de esto es la respuesta de Carmen Lyra a la publicación, en El Diario de Costa Rica en la cual se destaca el papel de la maestra Catalina Fallas para la llegada de una cañería a San Lorenzo de Tarrazú, ante esto Lyra escribe el artículo Catalina Fallas en el quincenario de pedagogía y otros estudios El Maestro, en el cual se une al reconocimiento y agrega que la escuela no es:

una estantería de libros pedantes y llenos de doctrinas, sino un centro de dinamismo, un anhelo de mejoramiento real de la humanidad. Y que si el programa pide que enseñe a sus alumnos que la fórmula del agua es $\mathrm{H} 2 \mathrm{O}$, la obligación de la Escuela que lo enseña es de velar porque se tome $\mathrm{H} 2 \mathrm{O}$ sin tricocéfalos ni amebas. (Lyra, 11 de agosto de 1926, p.23).

Un año más tarde, Lyra sería más explícita al denunciar a quienes ejercen la docencia desde un enfoque tradicional y reproduccionista, centrados en la academia y en la transmisión de contenidos al tiempo que ignoran, o al menos pretenden ignorar, las realidades de sus estudiantes y del país. Esto, en el marco del conflicto de límites con Panamá, afirmó:

los maestros, que deberíamos ser de vigías, no somos sino ratones de cueva. De la luz del día y de la oscuridad de la noche sobre los campos que transitamos, casi nada sabemos. En trasegar de los libros a las cabezas del niño o de los jóvenes, se nos va el tiempo. Y, entre tanto, la vida llora y ríe del otro lado de nuestra puerta cerrada. (Lyra, 15 de noviembre de 1927, p. 81).

Como parte de la segunda etapa (vinculación directa con los principios marxistas), Lyra (28 de julio de 1935) profundizaría el anterior señalamiento, al indicar:

a los maestros nos pasa en nuestro campo lo que a los campesinos agricultores en el suyo: que por estar siempre con la atención concentrada en sus cultivos no le dan importancia a los demás acontecimientos que se suceden a su alrededor; los maestros por estar pensando en lo van a enseñar, no ven ni meditan los fenómenos sociales que ocurren en torno suyo. Y si los ven, tienen que recortarlos para dejarlos a la medida de los intereses de la clase a que se sirven. (p. 3).

En ambos textos, Lyra insiste en la crítica al docente aislado de la realidad social, centrados en la transmisión de contenidos y quien asume a los estudiantes como objetos a los cuales hay llenar de conocimiento, sin embargo en el segundo de los textos incorpora la crítica a ese docente como formador de personas a la medida de los intereses de clase a que se sirven, esto como reflejo de la incorporación de los posicionamientos pedagógicos a las narraciones, aspiraciones y denuncia del Partido Comunista. Con esta breve incorporación, que sería profundizada en los próximos años, los pedagogos críticos del Partido Comunista construyen un eje de análisis donde "los intelectuales y maestros ocupamos un lugar definido, sea en las filas de la reacción, o sea en las filas del progreso, según como favorezcamos a las unas o a las otras con nuestra acción con nuestra inercia" (Sáenz, 4 de febrero de 1939, p. 3).

$\mathrm{Al}$ respecto, durante la Huelga Bananera de 1934 (principal movimiento sindical de la primera mitad del siglo XX), en el periódico Trabajo se publicó el artículo Los Maestros de Costa Rica no debe ilustrarse solo en la prensa burguesa sobre la buelga del Atlántico en el cual señalaba:

Quizá los maestros del tercero y sexto grado, se estén refiriendo en estos días en sus lecciones de Geografía, a los principales cultivos del país, a las relaciones comerciales de Costa Rica con otros países y a aquells (sic) productos que son la base de nuestra exportación, y como es natural, el banano habrá jugado un papel muy importante en tales conversaciones. Los maestros habrán enseñado a sus alumnos que el banano es una de las grandes riquezas de Costa Rica, que se cultiva en la zona atlántica y que ha comenzado a cultivarse en el Pacífico; que se exporta principalmente a los Estados Unidos, y los más enterados habrán dado el dato de los miles o millones de racimos que se exportaron el año pasado. Pero no habrán dicho quiénes son los verdaderos beneficiados con est (sic) riqueza costarricense ni le habrán prestado atención alguna al factor humano...De la vida miserable que llevan los peones que lo siembran, cuidan y cosechan, no se dice una palabra en la escuela, como tampoco de la rapacidad de la United Fruit Co. ni de la esclavitud a que ha reducido al pequeño productor (Anónimo, 19 de agosto de 1934, p. 3).

En este llamamiento, en Trabajo se asumía una premisa de la pedagogía crítica: la asociación de los contenidos a enseñar con problemáticas locales, regionales, nacionales y mundiales. La asociación trasciende 
lo circunstancial para convertirse en un llamado a la problematización del aprendizaje y a la concientización, tanto del docente como del estudiante, sobre explotación, la división de clases, la denuncia y visibilización de la burguesía y el imperialismo rapaz de la compañía United.

En línea con lo anterior, durante el primer quinquenio de la década de 1930 se profundiza la denuncia de la propuesta del proyecto hegemónico capitalista en Costa Rica y cómo el profesorado no

... goza de ninguna garantía y tiene que adoptar una actitud servil ante los procedimientos del gobierno. Si se atreve a criticar alguna actitud dudosa o torcida de un alto funcionario, es destituido, igualmente se extendían llamados para luchar por un aumento de sueldo (Anónimo, 1 de julio de 1934, p. 4).

\section{Por cuanto,}

Los idealistas de agua de azúcar, podrán encontrar que la lucha por mejoras de salarios, y por defensa de los derechos adquiridos, no cuadra con el apostolado del magisterio.

Nosotros en cambio encontramos que la miseria y el hambre no cuadran nunca con las más sabías reformas educativas (Anónimo, 27 de junio de 1936, p. 2.).

Con este texto, el periódico Trabajo y el Partido Comunista, zanjaban una división entre la concepción tradicional de docente en el marco de un proyecto hegemónico capitalista (capitalismo agrario en el caso costarricense) según el cual el docente emulaba las características de un sacerdote tanto en la misión (llevar a un rebaño de personas a los pastos previamente definidos en el proyecto católico, esto en el marco de una separación entre la misión y el contexto) como en las características materiales (ir donde fuese necesitado y mandado por la jerarquía, vida bajo el voto de pobreza y disciplina frente a la jerarquía). Por el contrario, el Partido Comunista y sus intelectuales pedagógicos sostenían que la miseria y el hambre no cuadran nunca con las más sabias reformas educativas, por tanto, se establece que el docente no es una extrapolación del sacerdocio, es un profesional que tiene una misión académica y social enmarcada por el compromiso de la transformación de sus realidades (laborales, profesionales, contexto de la escuela, familias, etc.) y de las realidades del país (revolución económica, social, cultural y política).

La correlación entre la mejora material de la calidad de vida del personal docente y una mejora educativa se reitera al afirmar en el artículo La cultura musical en las escuelas que "Sí, que se eleve la cultura musical en nuestras escuelas, pero también que a los maestros de música se les trate como a seres humanos. ¿Qué individuo puede estar capacitado para escuchar a Beethoven, si anda con el estómago vacío?" (Anónimo, 4 de junio de 1938, p. 3). En esta afirmación es implícito el precepto marxista de que la transformación de la situación material-económica es anterior a la transformación cultural-social, reiterado en el Discurso pronunciado en la CONVENCIÓN del Bloque de Obreros y Campesinos de Carlos Luis Sáenz (4 de febrero de 1939), en el cual indicó;

Queremos también que el maestro, no viva como un miserable; que su sueldo le alcance para vivir, no con lujo, pero sí con decencia; que tenga libertad de cátedra, es decir, que no le torne en un eunuco de su profesión. Queremos que esté garantizado en su puesto, por su moralidad y por su eficiencia y que su puesto no dependa del compadrazgo o de la buena o mala voluntad del gamonal ignorante. Queremos, en fin, un maestro libre en una escuela libre, para que en ella se eduque un pueblo libre (p. 3).

El texto anterior evidencia una profundización de la crítica y las asociaciones que elaboraban líderes de la pedagogía en el Partido Comunista, pues establecen las asociaciones entre la demanda de mejoras materiales con condiciones laborales como la libertad de cátedra y la estabilidad laboral, con la lógica de aspirar a "un maestro libre en una escuela libre, para que en ella se eduque un pueblo libre" (Sáenz, 4 de febrero de 1939, p. 3), asociaciones clásicas en la pedagogía crítica durante el siglo XX.

Los pedagogos críticos y las pedagogas críticas vincularon la demanda de trascender los límites del aula con el contexto económico costarricense (clases populares desposeídas y extrema desigualdad social) y los movimientos sociales que incrementaban la afiliación, temáticas y acciones post crisis económica de 1929, al respecto se afirmaba que el grave problema de la miseria en la escuela "tiene hondas raíces en el sistema 
político y económico que nos gobierna; por esto es absurdo que los maestros sean tan ajenos a los movimientos sociales que representan un anhelo de transformación en la vida" (Anónimo, 1932, p. 2).

En este nuevo análisis (marxista) el papel del educador anhelado por los pedagogos críticos está intrínsecamente vinculado su rol como promotor y actor de la transformación. Al respecto, la diferencia entre la concepción de educador y educadora entre movimientos pedagógicos progresistas y quienes enarbolan la pedagogía crítica ligada al Partido Comunista es que los primeros consideraban como centro de la acción el centro educativo, mientras que los segundos insistieron en la necesaria vinculación dialéctica entre cambios en los centros educativos y la acción para la transformación de la realidad, en tanto:

Hay educadores que creen de buena fé (sic) que es posible transformar la sociedad por medio de la influencia de la escuela. Pero esto de tomar como punto de partida para la transformación social nada más que al niño en la escuela es como querer construir una pared sobre el vacío. Solo a través de la lucha social serán posibles las verdaderas reformas en educación (Anónimo, 19 de marzo de 1933, p. 3).

En línea con lo anterior, Arnoldo Ferreto (1934) en su calidad de líder pedagógico del Partido Comunista junto a Lyra, González y Sáenz, sostuvo que para "el maestro revolucionario, para el maestro que dentro del régimen actual ve reducida su existencia a maldar(sic) lecciones, la revolución es, a la par que una necesidad material, una necesidad cultural” (p. 3). En este mensaje se incorpora la visión del profesorado revolucionario premisa fundamental de la pedagogía crítica e incorpora la necesidad de la revolución como necesidad material y cultural; el uso de este lenguaje demuestra mayor empoderamiento de los pedagogos críticos del Partido Comunista y menor limitación en el uso de reivindicaciones, concepciones y lenguaje que sería sancionado por los sectores de la oligarquía y el conservadurismo costarricense. La transformación del lenguaje implicó una polaridad entre educadores comprometidos con el bien de la nación y quienes estaban comprometidos con el capital, estos últimos

al servicio del capital, elaboran planes y programas sin conceptos definidos, planes y programas eclécticos, como dicen ellos para disimular su servilismo al régimen, es decir cogiendo de aquí y de allá lo que no pueda sembrar dudas en el ánimo de los alumnos, ni ofrezca el menor peligro para la seguridad del régimen capitalista (Anónimo, 19 de marzo de 1933, p. 3).

Con estas afirmaciones, en Trabajo se reiteraba la diferenciación entre los tipos de docentes, siendo las personas representantes de la pedagogía crítica ligadas al comunismo llamados a romper con la estructura educativa vertical y aislada, aquella donde no existe cuestionamiento social ni mayor objetivo que el académico. Para tal fin, es recurrente el uso de reflexiones en primera persona sobre la posibilidad de efectuar estos cambios, en este sentido Lyra (28 de julio de 1935), recordaba que, durante su paso en la Escuela Maternal, "no estaba encerrada en el molde de un programa cuyo fin es meter conocimientos más o menos abstractos en la inteligencia de los niños. Mis objetivos principales eran la alegría y la salud de los niños” (p. 3.)

Lo expuesto en este apartado permite establecer la construcción conceptual del educador o de la educadora por parte de los pedagogos críticos y pedagogas críticas ligados al Partido Comunista, pasando de una etapa de leves vinculaciones marxistas a un posicionamiento con principios marxistas.

Resulta evidente las conexiones entre educador-transformador tanto en aspectos del aula como en la vida nacional, así como la delimitación de educadores y educadoras que mantienen vínculos con el pensamiento crítico, consciente de las necesidades de las clases populares, promotor del cambio social y pedagógico y comprometido con una mejora de las condiciones materiales de los agentes del sistema educativo (salarios, pensiones y otros beneficios para docentes así como comedores escolares, libros, becas y salud para los niños y sus familias), siempre con la consigna de destruir el temor implantado en un sistema educativo sin condiciones de estabilidad laboral y sustentado en el temor (hacia las jerarquías por parte del docente y hacia los docentes y el aprendizaje por parte del estudiante), en el cual "A los alumnos los asustan con este coco de los exámenes y de las notas de concepto; a los profesores con este otro de echarlos del puesto” (Anónimo, 11 de julio de 1936, p. 5). 
Con el objetivo de comprender las implicaciones en la contextualización curricular de lo esbozado en apartados previos, se procederá con un análisis de la propuesta conceptual y operacional de la enseñanzaaprendizaje y del conocimiento construida paralelamente a la elaboración de los marcos de sistema educativoescuela y educador.

\section{ENSEÑAR Y APRENDER PARA EL CAMBIO ESTRUCTURAL}

El sistema educativo legado del siglo XIX enfrentaba, al iniciar el siglo XX, cuestionamientos estructurales tanto de progresistas como de conservadores. Se coincidía en la necesidad de transformar la metodología magistral, la inamovilidad del estudiante en su pupitre, la memorización aislada y asumir al estudiante como agente pasivo; todo esto para alcanzar una escuela activa pertinente a sociedades afectas por los rápidos cambios económicos, políticos, culturales, urbanísticos y sociales. Al respecto, Dengo (2009) afirmaba:

¿Por qué no recurrir al dibujo, por los niños, o al recortado de papel en aquella lección que utilizó figuras geométricas? Si los niños intervienen, es decir, colaboran de ese modo, se obtiene no solo un mayor interés en la lección, sino también un provechoso ejercicio de la actividad sensorial de ellos.

¿Por qué no asociar el dibujo a la lección sobre las plantas? Aplaudo lo de haber llevado flores, pero habría preferido que los niños hubiesen estado en mayor contacto con ellas en el momento de recoger observaciones....

Visto con los ojos de la escuela activa, el pupitre es un pequeño taller y un pequeño laboratorio. Dibujos, notas, recortes, etc., pueden suministrar los medios de que en ese taller y en ese laboratorio se forje la obra de un ser que puede hacer algo más que un oír y contestar: que puede pensar, que puede crear (p. 409).

En línea con la profundización del debate didáctico y específicamente sobre el uso de libro de textos, Dengo (2009), afirmó:

El problema viene a depender mucho de cómo sea el texto y de cómo se le use. De modo que, si llega a implantarlos, urge cuidarse de que no sean obligatorios y, sobre todo, de que no sean 'únicos'. Si es funesto que 'cada maestrito tenga su librito', es peor que cada alumno no lo tenga también. Hay que cuidarse también del uso que se va a hacer de tales libros. Con el mejor texto, el mal profesor convierte a los muchachos en papagayos, con todas las consecuencias que al respecto son bien conocidas (p. 417).

Se denota la preocupación de Dengo (2009), y en general de los pedagogos progresistas al iniciar el siglo XX, sobre el uso de libros de texto en primer lugar por el posible uso, sea obligatorio o elaborado por cada maestrito, en el tanto implicaría la reproducción del proyecto hegemónico (obligatorio nacional) o la implantación del currículum interpretado por el docente (elaborado por cada docente). Así mismo, se cuestiona la posibilidad de que cada estudiante pueda adquirirlo y, sobre todo, la posibilidad de que el uso del material simplemente reitere la pedagogía que convierte a los muchachos en papagayos, es decir, acumuladores y repetidores de contenidos sin comprensión de estos.

Sumado a lo anterior, Dengo (2009) afirmó que:

Cuando el aula pierde artificiosidad, cuando los temas no se originan en el capricho del maestro, cuando el objeto primordial no es el de calificar al niño, cuando se mueve este con independencia, cuando por todo ello es algo más que un inconsciente reproductor de las ideas del maestro, cuando no se le sujeta a la presión de un plan arbitrario, cuando, en suma, hay genuina motivación del trabajo, los ejercicios ascienden a un plano de realidad que se identifica con el de la vida (p. 415).

En esta reflexión, Dengo (2009) establece la ruptura con sistema de enseñanza tradicional como mecanismo para lograr que posteriormente el socioconstructivismo llamara aprendizaje significativo, es decir, el aprendizaje que logra sinergias con las realidades del estudiante, el centro educativo y la comunidad nacional. Así mismo, establece, por yuxtaposición narrativa, características de una nueva enseñanza que trascienda la evaluación como prioridad, la acción participativa e independiente del estudiante, la promoción del pensamiento crítico, la construcción colectiva del plan o programa de estudios y la motivación como principio de aprendizaje. 
La transformación de la enseñanza y el aprendizaje también era una tarea social, en el tanto:

Conocéis el castigo que consiste en obligar al niño a que deje el juego por el estudio. Ahí la más fuerte sugestión: el estudio, vehículo de la luz, convertido en tortura... ¿No es el juego, durante una edad, la escuela llena de alegría, distribuida en mil aulas, donde a todas horas hay trabajo y cada cosa da con encanto una lección de enseñanzas que para siempre sirven y son inolvidables? (Dengo, 2009, p. 346).

Por tanto, resultaba necesario el cambio de paradigma en los adultos y en la sociedad en general para comprender la importancia del juego y de las nuevas interacciones didácticas como mecanismo para construir aprendizajes relevantes, pertinentes, duraderos y significativos en el marco del contexto.

Otro cambio del paradigma de docentes, adultos y del sistema educativo en general fue sintetizado por Dengo (2009) cuando afirmó que:

Una mañana el niño entra a la escuela con la impaciencia de una pregunta que su curiosidad recogió en el trayecto. El maestro no la contesta. Afirma que no es la ocasión. El niño no puede expresar la inquisitiva actitud en que hay desilusión y sorpresa. ¿Por qué no se le contestó? El maestro había destinado la lección a tratar de la superficie del cilindro. En la mente del niño nada justifica la lección. ¿ De dónde sale el cilindro, qué importancia tiene, qué contacto con su interés, su vida? Si miráis con penetración hacia el aula, encontraréis que al niño se le contesta lo que nunca ha preguntado. Solo eso. Y que, en cambio, los resortes maravillosos de su actividad, capaces de lanzar sobre el mundo un tesoro de fuerza creadora de civilización, permanecen ocultos, intactos, tras la vacilante pregunta que el maestro no contestó (p. 350).

En esta narración se encuentran, implícitamente, los cuestionamientos de la pedagogía alternativa de la época y de la pedagogía crítica posteriormente desarrollada. En primer lugar, la reivindicación del estudiante como ser activo que construye conocimientos cotidianos y cuyo bagaje debe ser incorporado en la actividad educativa, en segundo lugar, se cuestiona la actitud del docente que minimiza e invisibiliza los temas construidos por el estudiante, en tercer lugar se denuncia a los programas oficiales que contesta lo que nunca ha preguntado el estudiante y, por último, la importancia de incorporar y aprovechar estas temáticas del estudiante para dinamizar el aprendizaje y la sociedad en general.

Los representantes de la pedagogía crítica asumieron las anteriores premisas de la enseñanza-aprendizaje y del conocimiento, incorporándolas en el contexto de necesidad de cambiar la realidad social, política y económica del país para consolidar un verdadero cambio educativo. Al respecto, en el caso de los conocimientos desarrollados en los centros educativos costarricenses durante la década de 1930, en Trabajo se afirmó:

Mal podrían los maestros sublevar a sus alumnos contra los explotadores: contarles, por ejemplo, cuando enseñan en geografía los productos del suelo en Costa Rica, cómo roban el trabajo del peón los grandes cafetaleros y cómo para que estos grandes cafetaleros tengan un beneficio del ciento por ciento, pagan jornales de hambre (Anónimo, 19 de marzo de 1933, p. 3).

En Trabajo se enunciaba una estrategia que sería recurrente en las reflexiones de los líderes y las lideresas pedagógicos del Partido Comunista: abrir espacios para la reflexión y el debate en el aula a partir del programa de estudio vigente. Así pues, en ninguna de las reflexiones estudiadas entre 1931 y 1948, se solicitó la incorporación o eliminación de contenidos del programa oficial de estudios, centrando los esfuerzos en la mediación del docente crítico para generar pensamiento alternativo o crítico en sus estudiantes a partir de los contenidos establecidos por el sistema y el proyecto hegemónico. Esto con el objetivo de que el estudiante debe "saber combatir, y debe saber construir y para eso es preciso que tenga conocimientos numerosos y profundos, que conozca y comprenda las últimas conquistas de la ciencia, que aprenda a servirse de lo que se sirve para construir" (Anónimo, 19 de marzo de 1933, p. 3).

La posición de representantes de la pedagogía crítica sobre la enseñanza-aprendizaje y conocimientos fue sintetizada por Carlos Luís Sáenz (7 de setiembre de 1940) bajo el título "Los colegios deben abordar con realismo el problema de la pubertad y del excesivo e inútil trabajo que EN FORMA DE TAREAS se IMPONE a los alumnos" (p. 3). En primer lugar, el autor destaca la formación pedagógica de los docentes de primaria contra la formación técnica-especializada de los docentes de secundaria, lo que favorece una primaria 
con métodos de enseñanza correlacionada, global y socialización, aspiraciones básicas del movimiento progresista y de la pedagogía crítica; no obstante, el autor omite señalar la formación en pensamiento crítico de los docentes de ambos ciclos educativos para implementar este cambio. Asimismo, establece el cambio del paradigma evaluativo para superar los exámenes tradicionales por test de apreciación y resultados "y en general una preocupación por parte de los maestros acerca del cómo y no del qué se enseña" (Sáenz, 7 de setiembre de 1940, p. 3).

En el caso de los docentes de secundaria, al ser especialista del área que ingresan al magisterio sin sustento pedagógico es "frecuente que ellos den sus lecciones como una mera copia del profesor que a ellos les enseñó la asignatura sin criterio ninguno con respecto a la metodología” (Sáenz, 7 de setiembre de 1940, p. 3).

Con esta premisa el autor insiste en un cuestionamiento crítico de la enseñanza tradicional y la reproducción que implica la introducción de docentes sin formación pedagógica y lo cual deriva en una educación "libresca y memorista, apegada a los métodos de estudio de resúmenes y textos, que solo tienen por finalidad ser repetidos por el alumno en los exámenes bimestrales o en los exámenes finales” (Sáenz, 7 de setiembre de 1940, p. 3), en algunos casos estos materiales son desarrollados por el personal educador y utilizados durante años ignorando los avances científicos y seleccionados por criterio de cada docente.

Además, implícitamente se cuestiona la estructuración del conocimiento desde el positivismo al afirmar que "Cada profesor trabaja por aparte sin tomar en cuenta los trabajos de los demás ni las vinculaciones culturales" (Sáenz, 7 de setiembre de 1940, p. 3) y con la afirmación de que el trabajo en la aulas "es generalmente o predominantemente individualista, propio para estimular el más cerrado egoísmo, no se enseña a los alumnos a trabajar colectivamente" (Sáenz, 7 de setiembre de 1940, p. 3) se señala directamente la ideología capitalista determinada en el currículum oculto de los centros educativos e incluso señala el cuestionamiento a la excelencia promovida bajo el sistema tradicional-capitalista como "el fetichismo de la alumna o del alumno de UNO CORRIDO como la máxima expresión del éxito en los estudiantes, lo cual revela un desconocimiento alarmante de la psicologa (sic) individual” (Sáenz, 7 de setiembre de 1940, p. 3).

Por si fuera poco, el autor señala el cuestionamiento a la función policial de los inspectores y "de cómo el estudiante se le echa fuera de clase, o se le expulsa o se le pone mala nota como sanciones que no remedian nada, puesto que son castigos externos que no logran mejorar al alumno en ninguna forma" (Sáenz, 7 de setiembre de 1940, p. 3), es decir, el autor en 1940 propuso una agenda de reforma de la evaluación de la conducta que no entraría al debate nacional hasta el año 2008. Caso similar sucede con la solicitud de "que el profesorado debe escogerse con más criterio científico, que el profesor debía por lo menos haber practicado algunos años en la primaria" (Sáenz, 7 de setiembre de 1940, p. 3), donde el trasfondo de la discusión radica en el sistema y perfil de contratación de los docentes.

Por último, el autor establece la necesidad de "abordar con realismo el problema de la pubertad y del excesivo trabajo que en forma de tareas inútiles y agotadoras impone a sus alumnos" (Sáenz, 7 de setiembre de 1940, p. 3); incorporando aspectos de la atención integral al estudiantado (atención de las características físico-emocionales de la pubertad) y cuestionando el régimen académico inútil y agotador producto de una visión tradicional, hegemónica y capitalista; en la lógica de un currículum técnico y reproductor en donde la acumulación de conocimientos era la prioridad.

\section{Conclusiones}

El ensayo permite establecer la presencia de un movimiento pedagógico liderado por Carmen Lyra, Carlos Luis Sáenz, Luisa González y Arnoldo Ferreto que funda, en la década de 1930 y de forma implícitamente, la pedagogía crítica costarricense desde el Partido Comunista.

El estudio del Diario Quincenal El Maestro (1926-1930), el Periódico Trabajo (1931-1948) y textos literarios de las personas autoras, permite establecer una diferenciación del discurso pedagógico previo a la fundación del Partido Comunista y después de este hecho, en la primera etapa se construye una narración 
focalizada en problemas cotidianos y la correspondiente exigencia de mejoras mientras que en la segunda etapa de elabora una profundización de la denuncia, las propuestas de transformación y la vinculación de ambas con las premisas comunistas. Es importante señalar que, durante la primera etapa, se construye una conceptualización educativa vinculada con: ejercicio educativo más allá de los contenidos, necesidad de romper con el aislamiento y pasividad del magisterio y vinculación de la educación con las necesidades de los niños y las niñas, centrado prioritariamente en los discursos de higiene y alimentación.

Los discursos vinculan la pedagogía, sociedad y reformas educativas desde una relación dialéctica y con una denuncia del silencio de docentes-grupos dominantes ligados a la pedagogía tradicional en los cuales se ignora el contexto y las necesidades de los hijos de los trabajadores o se les reduce a estadísticas y se les condena como agentes de enfermedades, vulgaridades y delincuencia. Adicionalmente, se asume la bandera de invertir en educación más allá de la apertura de centros educativos, pues se establece la necesidad de invertir en útiles escolares, alimentación y vestido para los estudiantes así como salarios y pensiones justas, sin obviar posturas progresistas en las temáticas de sexualidad, laicismo, educación democrática y la creación del Consejo Nacional de Educación como forma para establecer política educativa de mediano plazo e independencia del sistema educativo con respecto al poder político transitorio.

La propuesta de reformas educativas se visibilizó en seis agendas educativas divulgadas por el Partido Comunista entre 1934 y 1948, las cuales aumentan en cantidad (de 5 puntos en 1934 a 15 en 1947) y en agendas temáticas pero en las cuales es clara la concepción de la educación y el centro educativo en interacción dialéctica con la sociedad, que debía luchar contra el proyecto político fascista y la dominación económica de la burguesía agraria mediante el uso de conocimientos contextual, un papel protagónico del educador como agente de reflexivo y de cambio y la inclusión del estudiantado pobre mediante políticas de seguridad social (alimentación, becas, construcción de colegios y otros).

En el marco de lo anterior, se exigía una nueva persona educadora que supere la enseñanza centrada en la transmisión de contenidos, impulsor de la vinculación aula-comunidad, consciente de las realidades socioeconómicas y políticas, luchador por la transformación de las desigualdades sociales, capaz de construir pensamiento crítico desde el currículo oficial utilizando contenidos del programa para problematizar y consciente del papel ideológico y práctico del sistema educativo dentro del modelo capitalista. Al mismo tiempo consciente de su rol de clase y su deber de luchar para transformar su situación laboral (inestabilidad, despidos, bajos salarios, ausencia de un régimen estable de pensiones y otros) bajo la premisa de que un mejor sistema educativo solo podría construirse sobre los cimientos de un docente dignificado y estudiantes con situaciones socioeconómicas mínimas (vivienda, higiene, alimentos, vestido y otros).

La nueva educación, y el nuevo educador, construyen un nuevo sistema de enseñanza y aprendizaje alejado del reproduccionismo tradicional para alcanzar el cambio estructural. La prioridad no debe ser aprender el contenido dictado por el programa oficial ni el uso repetitivo de libros o manuales, sino aprender activamente y correlacionadamente en el contexto del estudiante, con el uso del juego, aprendiendo para la vida y a partir de las preguntas generadoras del estudiante en su cotidianidad, todo esto para lograr que el estudiante asuma un papel crítico y de transformación.

Por tanto, es visible las interacciones entre las demandas del movimiento pedagógico progresista ligado con el Partido Comunista y la agenda de transformación educativa de la pedagogía crítica, en lo que podría denominarse las raíces de la pedagogía crítica costarricense.

\section{REFERENCIAS}

Anónimo, (10 de octubre de 1931). Notas de la redacción. Trabajo, pp. 1, 4.

Anónimo, (13 de marzo de 1932a). Primer Programa Mínimo del Partido Comunista. Trabajo, p. 2.

Anónimo, (13 de marzo de 1932b). Los hijos de los trabajadores. Trabajo, pp. 2-4.

Anónimo, (19 de marzo de 1933). Actitud del congreso frente al presupuesto en educación. Trabajo, p. 3. 
Anónimo, (4 de febrero de 1934). Conquistas inmediatas que para los maestros propone el Bloque de Obreros y Campesinos. Trabajo, p. 4

Anónimo, (1 de julio de 1934). Que se les aumente los sueldos a los maestros. Trabajo, p. 4.

Anónimo, (19 de agosto de 1934). Los Maestros de Costa Rica no debe ilustrarse solo en la prensa burguesa sobre la huelga del Atlántico, Trabajo, p. 3

Anónimo, (7 de octubre de 1934). El congreso Pedagógico. Trabajo, p. 1.

Anónimo, (14 de abril de 1935). La disciplina militarista implantada por Picado en las escuelas, ya está dando sus frutos. Trabajo, p. 1

Anónimo, (27 de junio de 1936). Los maestros deben pensar en organizarse seriamente. Trabajo, p. 2.

Anónimo, (11 de julio de 1936). Triste espectáculo de nuestra educación. Trabajo, p. 5.

Anónimo, (30 de enero de 1938). Para defender los intereses del pueblo no basta ser una buena persona. Trabajo, p. 3-4 Anónimo, (4 de junio de 1938). La cultura musical en las escuelas. Trabajo, p. 3.

Anónimo, (15 de septiembre de 1938). El cincuentenario de la fundación del Colegio de Señoritas y el Congreso Centroamericano Femenino de Educación. Trabajo, p. 2.

Anónimo, (14 de junio de 1941). Primer Programa Mínimo del Partido Comunista. Trabajo, p. 2.

Anónimo, (10 de enero de 1942). Aquí está nuestro PROGRAMA. Trabajo, pp. 3-4

Anónimo, (26 de junio de 1943). Programa de Vanguardia Popular. Trabajo, p. 3

Anónimo, (20 de enero de 1945). Enseñanza Secundaria gratuita para los estudiantes pobres. Trabajo, p. 1.

Anónimo, (20 de febrero de 1946) Programa del Bloque de la Victoria. Trabajo, p. 4.

Dengo, O. (2009). Escritos y discursos. Heredia: EUNA.

Ferreto, A. (8 de julio de 1934). El niño y la escuela en Costa Rica. Trabajo, p. 3.

González, L. y Saénz, C. L. (1977). Carmen Lyra. San José, Costa Rica: Ministerio de Cultura, Juventud y Deportes

Lyra, C. (11 de agosto de 1926). Catalina Fallas. El Maestro, p. 23.

Lyra, C. (15 de noviembre de 1927). Comentarios. El Maestro, 81-82

Lyra, C. (28 de julio de 1935). El régimen capitalista es el pasado. El comunismo cuenta con el futuro y el presente también está de su parte. Fragmentos de la conferencia radiodifundida el 22 de este mes por nuestra c. Carmen Lyra desde la estación Ecos de Occidente. Trabajo, p. 3

Lyra, C. (27 de mayo de 1939). Carmen Lyra hace una brillante defensa a los maestros. Trabajo, p. 4

Molina, I. (2000). La ciudad de los monos. Heredia: EUNA.

Sáenz, C. (4 de febrero de 1939). Discurso pronunciado en la CONVENCIÓN del Bloque de Obreros y Campesinos, el lunes 30 de enero. Trabajo, p. 3

Sáenz, C. (7 de setiembre de 1940). Los colegios deben abordar con realismo el problema de la pubertad y del excesivo e inútil trabajo que EN FORMA DE TAREAS se IMPONE a los alumnos. Trabajo, p. 3.

Sáenz, C. (15 de septiembre de 1940). La Escuela y la Democracia en nuestro país. Trabajo, pp. 6-7.

\section{BY-NC-ND}

